\title{
Pralatrexate Regimen
}

National Cancer Institute

\section{Source}

National Cancer Institute. Pralatrexate Regimen. NCI Thesaurus. Code C160810.

A regimen consisting of pralatrexate that may be used in the treatment of relapsed or refractory peripheral T-cell lymphoma (PTCL). 